\title{
Efficient Synthesis of Novel 3-Substituted Coumarin-3-carboxamide
}

\author{
Enayatollah Sheikhhosseini, Saeed Balalaie ${ }^{\dagger, *}$, Mohammad Ali Bigdeli, \\ Azizollah Habibi ${ }^{\ddagger}$, and Hamed Piri Moghaddam ${ }^{\S}$ \\ Department of Chemistry, Faculty of Science, Kerman Branch, Islamic Azad University, Kerman, Iran \\ ${ }^{\dagger}$ Peptide Chemistry Research Center, K. N. Toosi University of Technology, P. O. Box 15875-4416, Tehran, Iran \\ *E-mail: balalaie@kntu.ac.ir \\ ${ }^{\star}$ Faculty of Chemistry, Kharazmi University, No. 49, Mofateh Ave. Tehran, Iran \\ ${ }^{\S}$ Department of Chemistry, Sharif University of Technology, Tehran, Iran \\ (Received October 13, 2013; Accepted January 22, 2014)
}

\begin{abstract}
A series of novel pseudopeptides contained coumarin skeleton were synthesized through the Ugi-four-component reaction. The 3-substituted coumarin-3-carboxamides were formed through reaction of benzaldehyde derivatives, anilines, coumarin-3carboxylic acid and isocyanides with high yields and high bond-forming efficiency at room temperature. These novel amidated coumarins exhibit brilliant fluorescence in range of 535-547 $\mathrm{nm}$ in chloroform.
\end{abstract}

Key words: 3-Substituted amidated coumarins, Ugi-4CR, Pseudopeptides, Fluoresence property

\section{INTRODUCTION}

The synthesis of coumarins and their derivatives has attracted considerable attention from organic and medicinal chemists for many years as a large number of natural products contain these heterocyclic molecules. ${ }^{1}$ They are widely used as additives in food, perfumes, cosmetics, pharmaceutical, ${ }^{2,3}$ optical brighteners ${ }^{4}$ and dispersed fluorescent and laser dyes. ${ }^{5}$ Natural coumarins and their synthetic structural analogs have broad biological activities, such as antimicrobial and antimicrobial, ${ }^{6}$ antitumor, ${ }^{7}$ and antiviral activities. ${ }^{8}$ Meanwhile, some coumarin derivatives could affect human immunodeciency virus integrase inhibitors. ${ }^{9}$ Some coumarins have inhibitory activity against some serine proteases and matrix metalloproteases (MMPs) ${ }^{10}$ and also act as a selective anti-proliferative agent. ${ }^{11-14}$

It is shown that the existence of amide functional groups in the structure of coumarins could affect the biological activities of these compounds and it is related to the patern of substitution and also its position in coumarin ring. ${ }^{15}$ According to these results, the number of amide bonds and also their position in the structure of coumarin scaffold could affect the type of biological activity. ${ }^{16-20}$

Meanwhile, coumarins are the largest class of laser dyes and highly sensitive for the blue-green region, include commercially available fluorescent derivatives of extended spectral range, high emission quantum yield, photostability and good solubility in many solvents. ${ }^{21,22}$ Recently, Katritzky and co-workers reported synthesis of fluorescent coumarin- aminoxy acid and coumarin-thioester which could be used for the detection of peptides. ${ }^{23,24}$

Based on the diverse biological activities of coumarins and their fluorescence property, the synthesis of compounds which contained more amide bonds is an interesting area in organic synthesis.

Multi-component reactions (MCRs) have been investigated extensively in organic and diversity-oriented synthesis; primarily due to their ability to generate complex molecular functionality from simple starting materials via one-pot reactions. ${ }^{25,26}$ For this reason, MCRs are featured in many diversity-oriented projects, whose biological relevance has been validated by the discovery of novel biological probes and drug leads. ${ }^{27}$ Due to importance of chromene scaffold, recently, Che et al. reported synthesis of chromeno [3,4c]pyrrole-3,4-diones via sequential Ugi/intramolecularMichael addition reactions. ${ }^{28}$

In continuation of our research work to design novel multicomponent reactions, ${ }^{29}$ we wish to report a simple one-pot four-component reaction of aromatic aldehydes (1), aniline (2), coumarin-3-carboxylic acid (3) and isocyanide (4) which leading to pseudopeptides which contained coumarin skeleton $\mathbf{5 a}-\mathbf{p}$ (Scheme 1).

\section{EXPERIMENTAL}

Commercially available materials were used without further purification. Melting points were determined on an Electrothermal 9100 apparatus and were uncorrected. 


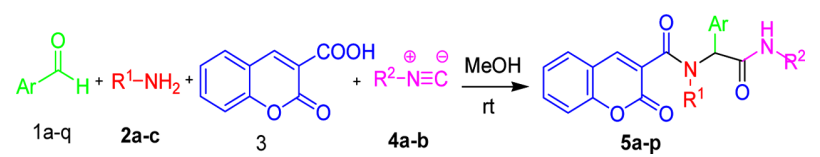

Scheme 1. Synthesis of 3-substituted coumarin carboxamides.

IR spectra were obtained on an ABB FT-IR FTLA 2000 spectrometer. ${ }^{1} \mathrm{H}$ NMR and ${ }^{13} \mathrm{C}$ NMR spectra were run on Bruker DRX-300 AVANCE spectrometer at $300 \mathrm{MHz}$ for ${ }^{1} \mathrm{H} \mathrm{NMR}$, and $75 \mathrm{MHz}$ for ${ }^{13} \mathrm{C} \mathrm{NMR}$. $\mathrm{CDCl}_{3}$ was used as solvent. HRMS was recorded on Mass-ESI-POS (Apex Qe-FT-ICR instrument) spectrometer. Fluorescence spectra for all compounds were collected at room temperature with a Photon Technology international model MP1 steadystate fluorimeter. Fluorescence measurements were taken using NSG Precision Cells (Farmingdale, NY) ES quartz cuvettes (190-2000 nm).

\section{Synthesis of Coumarin-3-carboxylic Acid}

In a round-bottomed flask, salicylaldehyde $(10 \mathrm{mmol})$ and Meldrum's acid $(12 \mathrm{mmol})$ in water $(20 \mathrm{ml})$ were heated at reflux under stirring for $10 \mathrm{~h}$. The reaction mixture was cooled and filtered on Büchner funnel. Further purification was done using crystallization in methanol (Yield = $95 \%$ ).

General Procedure for the Synthesis of Chromene-3carboxamide Derivatives (5a-q)

Primary amine 2 (1 mmol) was added to a solution of aldehyde 1 (1 mmol) in methanol $(5 \mathrm{~mL})$ and the reaction mixture was stirred at room temperature for $1 \mathrm{~h}$. Coumarin3-carboxylic acid 3 ( $1 \mathrm{mmol}$ ) was added and stirring was continued for $15 \mathrm{~min}$ followed by addition of isocyanide 4 ( $1 \mathrm{mmol})$. The resulting solution was stirred at room temperature for 4-6h. The reaction was complete after 4-6h. The solvent was removed under reduced pressure and the product was precipitated by addition of water. Precipitated solid product was recrystallized from water/acetone and identified (Yields 77-93\%).

N-((Cyclohexylcarbamoyl)(phenyl)methyl)-2-oxo-N-phenyl-2H-chromone-3-carboxamide (5a): Yield 90\%. Mp $=233-236{ }^{\circ} \mathrm{C} . \mathrm{IR}\left(\mathrm{KBr}, \mathrm{cm}^{-1}\right): 3262,1717,1649,1641 \mathrm{~cm}^{-1}$. ${ }^{1} \mathrm{HNMR}\left(300 \mathrm{MHz}, \mathrm{CDCl}_{3}\right): \delta=1.15-2.08\left(\mathrm{~m}, 10 \mathrm{H}, 5 \mathrm{CH}_{2}\right)$, $3.93(\mathrm{~m}, 1 \mathrm{H}, \mathrm{CH}-\mathrm{NH}), 6.35$ (s, 1H, CH-N), 6.78 (br s, 1H, NH), 7.00-7.50 (14H, m, H-Ar), 7.80 (s, 1H, =CH). ${ }^{13} \mathrm{C} \mathrm{NMR}\left(75 \mathrm{MHz}, \mathrm{CDCl}_{3}\right) \delta: 24.8,24.9,25.4,32.7,33.0$, 49.1, 65.7, 116.7, 117.9, 124.8, 125.9, 128.1, 128.2, 128.4, 128.5, 129.9, 130.5, 132.5, 134.0, 138.8, 142.3, 153.6, 158.3, 165.4, 167.8. HR-MS (ESI) calcd for $\mathrm{C}_{30} \mathrm{H}_{29} \mathrm{~N}_{2} \mathrm{O}_{4}[\mathrm{M}+\mathrm{H}]^{+}$
481.21249, found 481.21246; calcd for $\mathrm{C}_{30} \mathrm{H}_{28} \mathrm{~N}_{2} \mathrm{NaO}_{4}$ $[\mathrm{M}+\mathrm{Na}]^{+}$503.19439, found 503.19442; calcd for $\mathrm{C}_{30} \mathrm{H}_{28} \mathrm{KN}_{2} \mathrm{O}_{4}$ $[\mathrm{M}+\mathrm{K}]^{+} 519.16828$, found 519.16830 .

N-((Cyclohexylcarbamoyl)(2-methoxyphenyl)methyl)-2oxo-N-phenyl-2H-chromone-3-carboxamide (5b): Yield $87 \% . \mathrm{Mp}=132-136^{\circ} \mathrm{C} . \mathrm{IR}\left(\mathrm{KBr}, \mathrm{cm}^{-1}\right): 3293,1726,1656$, $1607 \mathrm{~cm}^{-1} .{ }^{1} \mathrm{H}$ NMR $\left(300 \mathrm{MHz}, \mathrm{CDCl}_{3}\right): \delta=1.21-2.15$ $\left(\mathrm{m}, 10 \mathrm{H}, 5 \mathrm{CH}_{2}\right), 3.75$ (s, 3H, OMe), 3.97 (m, 1H, CH$\mathrm{NH}), 6.60(\mathrm{~s}, 1 \mathrm{H}, \mathrm{CH}-\mathrm{N}), 6.65(\mathrm{~d}, 1 \mathrm{H}, J=8.1 \mathrm{~Hz}, \mathrm{H}-\mathrm{Ar})$, $6.73(\mathrm{t}, 1 \mathrm{H}, J=7.5 \mathrm{~Hz}, \mathrm{H}-\mathrm{Ar}), 6.90-7.51$ (m, 12H, NH and $\mathrm{H}-\mathrm{Ar}), 7.83(\mathrm{~s}, 1 \mathrm{H},=\mathrm{CH}) .{ }^{13} \mathrm{C} \mathrm{NMR}\left(75 \mathrm{MHz}, \mathrm{CDCl}_{3}\right) \delta$ : 24.9, 25.1, 25.5, 32.7, 33.2, 48.9, 55.1, 60.4, 110.0, 116.7, $118.1,120.2,122.4,124.8,126.3,127.8,128.0,128.4,129.6$, 129.8, 131.4, 132.4, 1387.7, 142.1, 153.6, 157.4, 158.4, 165.3, 168.4. HR-MS (ESI) calcd for $\mathrm{C}_{31} \mathrm{H}_{31} \mathrm{~N}_{2} \mathrm{O}_{5}[\mathrm{M}+\mathrm{H}]^{+}$ 511.22292, found 511.22290; calcd for $\mathrm{C}_{31} \mathrm{H}_{30} \mathrm{~N}_{2} \mathrm{NaO}_{5}$ $[\mathrm{M}+\mathrm{Na}]^{+}$533.20485, found 533.20484; calcd for $\mathrm{C}_{31} \mathrm{H}_{30} \mathrm{KN}_{2} \mathrm{O}_{5}$ $[\mathrm{M}+\mathrm{K}]^{+}$549.17867, found 549.17867.

N-((Cyclohexylcarbamoyl)(4-methoxyphenyl)methyl)-2oxo-N-phenyl-2H-chromone-3-carboxamide (5c): Yield $92 \% . \mathrm{Mp}=202-205^{\circ} \mathrm{C} . \mathrm{IR}\left(\mathrm{KBr}, \mathrm{cm}^{-1}\right): 3271,1720,1656$, $1609 \mathrm{~cm}^{-1} .{ }^{1} \mathrm{H}$ NMR $\left(300 \mathrm{MHz}, \mathrm{CDCl}_{3}\right): \delta=1.16-2.06(\mathrm{~m}$, $\left.10 \mathrm{H}, 5 \mathrm{CH}_{2}\right), 3.71$ (s, 3H, OMe), $3.92(\mathrm{~m}, 1 \mathrm{H}, \mathrm{CH}-\mathrm{NH})$, $6.29(\mathrm{~s}, 1 \mathrm{H}, \mathrm{CH}-\mathrm{N}), 6.66$ (br s, $1 \mathrm{H}, \mathrm{NH}), 6.67$ (d, $J=8.6$ $\mathrm{Hz}, 2 \mathrm{H}, \mathrm{H}-\mathrm{Ar}$ ), 7.07 (d, J=8.6 Hz, 2H, H-Ar), 7.02-7.49 (m, 9H, H-Ar) $7.78(\mathrm{~s}, 1 \mathrm{H},=\mathrm{CH}) .{ }^{13} \mathrm{C} \mathrm{NMR}(75 \mathrm{MHz}$, $\left.\mathrm{CDCl}_{3}\right) \delta: 24.8,24.9,25.4,32.7,32.9,49.0,55.1,65.0$, 113.6, 116.6, 117.9, 124.7, 125.9, 126.0, 128.1, 128.4, 128.5, $130.0,131.8,132.5,138.8,142.1,153.6,158.2,159.3$, 165.3, 168.2. HR-MS (ESI) calcd for $\mathrm{C}_{31} \mathrm{H}_{31} \mathrm{~N}_{2} \mathrm{O}_{5}[\mathrm{M}+\mathrm{H}]^{+}$ 511.22277, found 511.22277; calcd for $\mathrm{C}_{31} \mathrm{H}_{30} \mathrm{~N}_{2} \mathrm{NaO}_{5}$ $[\mathrm{M}+\mathrm{Na}]^{+}$533.20471, found 533.20471; calcd for $\mathrm{C}_{31} \mathrm{H}_{30} \mathrm{KN}_{2} \mathrm{O}_{5}$ $[\mathrm{M}+\mathrm{K}]^{+}$549.17862, found 549.17862.

N-((Cyclohexylcarbamoyl)(2,4-dimethoxyphenyl)methyl)2-oxo-N-phenyl-2H-chromone-3-carboxamide (5d): Yield $93 \% . \mathrm{Mp}=189-192{ }^{\circ} \mathrm{C} . \mathrm{IR}\left(\mathrm{KBr}, \mathrm{cm}^{-1}\right): 3274,1718,1654$, $1607 \mathrm{~cm}^{-1}$. ${ }^{1} \mathrm{H}$ NMR $\left(300 \mathrm{MHz}, \mathrm{CDCl}_{3}\right): \delta=1.19-2.08(\mathrm{~m}$, $\left.10 \mathrm{H}, 5 \mathrm{CH}_{2}\right), 3.69$ (s, 6H, 2OMe), $3.94(\mathrm{~m}, 1 \mathrm{H}, \mathrm{CH}-\mathrm{NH})$, 6.20 (d, 1H, $J=2.4 \mathrm{~Hz}, \mathrm{H}-\mathrm{Ar}), 6.24-6.27(\mathrm{dd}, 1 \mathrm{H}, J=7.8$, $2.4 \mathrm{~Hz}, \mathrm{H}-\mathrm{Ar}), 6.50$ (s, 1H, CH-N), 6.82 (br s, 1H, NH), 6.83-7.20 (7H, m, H-Ar), 7.40-7.50 (m, 2H, H-Ar), 7.79 $(1 \mathrm{H}, \mathrm{s},=\mathrm{CH}) .{ }^{13} \mathrm{C} \mathrm{NMR}\left(75 \mathrm{MHz}, \mathrm{CDCl}_{3}\right) \delta: 24.9,25.1$, 25.5, 32.7, 33.2, 48.9, 55.1, 55.2, 60.2, 97.9, 104.0, 114.8, 116.7, 118.1, 124.7, 126.3, 127.8, 128.0, 28.4, 129.7, 132.1, $132.3,138.8,141.9,153.6,158.5,161.0,165.3,168.6$. HR-MS (ESI) calcd for $\mathrm{C}_{32} \mathrm{H}_{33} \mathrm{~N}_{2} \mathrm{O}_{6}[\mathrm{M}+\mathrm{H}]^{+}$541.23498, found 541.23483; calcd for $\mathrm{C}_{32} \mathrm{H}_{32} \mathrm{~N}_{2} \mathrm{NaO}_{6}[\mathrm{M}+\mathrm{Na}]^{+}$563.21672, found 563.21698; calcd for $\mathrm{C}_{32} \mathrm{H}_{32} \mathrm{KN}_{2} \mathrm{O}_{6}[\mathrm{M}+\mathrm{K}]^{+} 579.19082$, found 579.19067 . 
$\mathrm{N}$-((Cyclohexylcarbamoyl)(3,4,5-trimethoxyphenyl)methyl)2-oxo-N-phenyl-2H-chromone-3-carboxamide (5e): Yield $88 \% . \mathrm{Mp}=168-170{ }^{\circ} \mathrm{C} . \mathrm{IR}\left(\mathrm{KBr}, \mathrm{cm}^{-1}\right): 3270,1718,1653$, $1606 \mathrm{~cm}^{-1} .{ }^{1} \mathrm{H} \mathrm{NMR}\left(300 \mathrm{MHz}, \mathrm{CDCl}_{3}\right): \delta=1.17-2.05(\mathrm{~m}$, $\left.10 \mathrm{H}, 5 \mathrm{CH}_{2}\right), 3.68$ (s, 6H, 2OMe), 3.75 (s, 3H, OMe), 3.90 (m, 1H, CH-NH), 6.21 (s, 1H, CH-N), 6.41 (s, 2H, H-Ar), 6.62 (br s, 1H, NH), 7.06-7.50 (m, 9H, H-Ar), 7.77 (s, $1 \mathrm{H},=\mathrm{CH}) .{ }^{13} \mathrm{CNMR}\left(75 \mathrm{MHz}, \mathrm{CDCl}_{3}\right) \delta: 24.8,24.9,25.4$, 32.7, 32.9, 49.0, 56.0, 60.7, 65.8, 107.6, 116.7, 117.9, 124.8, $125.8,128.3,128.4,128.6,129.2,129.9,132.6,137.8,138.9$, 142.1, 152.8, 153.6, 158.3, 165.4, 167.8. HR-MS (ESI) calcd for $\mathrm{C}_{33} \mathrm{H}_{35} \mathrm{~N}_{2} \mathrm{O}_{7}[\mathrm{M}+\mathrm{H}]^{+}$571.24557, found 571.24542; calcd for $\mathrm{C}_{33} \mathrm{H}_{34} \mathrm{~N}_{2} \mathrm{NaO}_{7}[\mathrm{M}+\mathrm{Na}]^{+}$593.22723, found 593.22710.

N-((Cyclohexylcarbamoyl)(p-tolyl)methyl)-2-oxo-N-phenyl-2H-chromone-3-carboxamide (5f): Yield 83\%. Mp = 220-222 ${ }^{\circ} \mathrm{C}$. IR $\left(\mathrm{KBr}, \mathrm{cm}^{-1}\right): 3314,1731,1655 \mathrm{~cm}^{-1} .{ }^{1} \mathrm{HNMR}$ $\left(300 \mathrm{MHz}, \mathrm{CDCl}_{3}\right): \delta=1.15-2.03\left(\mathrm{~m}, 10 \mathrm{H}, 5 \mathrm{CH}_{2}\right), 2.24$ (s, 3H, Me), $3.92(\mathrm{~m}, 1 \mathrm{H}, \mathrm{CH}-\mathrm{NH}), 6.27$ (s, 1H, CH-N), 6.59 (br s, 1H, NH), 6.90-7.25 (m, 11H, H-Ar), 7.41 (d, $1 \mathrm{H}, J=7.5 \mathrm{~Hz}, \mathrm{H}-\mathrm{Ar}), 7.47$ (t, $1 \mathrm{H}, J=7.5 \mathrm{~Hz}, \mathrm{H}-\mathrm{Ar}), 7.78$ $(\mathrm{s}, 1 \mathrm{H},=\mathrm{CH}) .{ }^{13} \mathrm{C} \mathrm{NMR}\left(75 \mathrm{MHz}, \mathrm{CDCl}_{3}\right) \delta: 21.1,24.9$, 25.0, 25.5, 32.8, 33.0, 49.0, 65.6, 116.7, 118.0, 124.8, 128.1, 128.2, 128.4, 128.5, 129.0, 130.0, 130.4, 130.9, 132.5, 138.1, 139.0, 142.2, 153.6, 165.8, 168.1. HR-MS (ESI) calcd for $\mathrm{C}_{31} \mathrm{H}_{31} \mathrm{~N}_{2} \mathrm{O}_{4}[\mathrm{M}+\mathrm{H}]^{+}$495.22857, found 495.22850; calcd for $\mathrm{C}_{31} \mathrm{H}_{30} \mathrm{~N}_{2} \mathrm{NaO}_{4}[\mathrm{M}+\mathrm{Na}]^{+}$517.21052, found 517.21045; calcd for $\mathrm{C}_{31} \mathrm{H}_{30} \mathrm{KN}_{2} \mathrm{O}_{4}[\mathrm{M}+\mathrm{K}]^{+}$533.18455, found 533.18447.

N-((Cyclohexylcarbamoyl)(4-isopropyl)methyl)-2-oxoN-phenyl-2H-chromone-3-carboxamide (5g): Yield 91\%. $\mathrm{Mp}=164-166^{\circ} \mathrm{C} . \mathrm{IR}\left(\mathrm{KBr}, \mathrm{cm}^{-1}\right): 33.18,1730,1653 \mathrm{~cm}^{-1}$. ${ }^{1} \mathrm{H} \mathrm{NMR}\left(300 \mathrm{MHz}, \mathrm{CDCl}_{3}\right): \delta=1.15(\mathrm{~d}, J=6.9 \mathrm{~Hz}, 6 \mathrm{H}$, 2Me), 1.19-2.07 (m, 10H, 5CH $), 2.79(\mathrm{~m}, 1 \mathrm{H}, J=6.90$ $\left.\mathrm{Hz}, \mathrm{CH}(\mathrm{Me})_{2}\right), 3.93$ (m, 1H, CH-NH), 6.27 (s, 1H, CH-N), 6.64 (br s, 1H, NH), 7.01-7.50 (m, 13H, H-Ar) 7.78 (s, 1H, $=\mathrm{CH}) .{ }^{13} \mathrm{C} \mathrm{NMR}\left(75 \mathrm{MHz}, \mathrm{CDCl}_{3}\right) \delta: 23.7,24.9,25.0,25.5$, 32.7, 32.9, 33.6, 49.0, 65.7, 116.7, 118.0, 124.7, 126.0, 126.3, 127.9, 128.4, 128.5, 129.9, 130.4, 131.2, 132.4, 139.0, 142.1, 149.0, 153.6, 158.2, 165.3, 168.1. HR-MS (ESI) calcd for $\mathrm{C}_{33} \mathrm{H}_{35} \mathrm{~N}_{2} \mathrm{O}_{4}[\mathrm{M}+\mathrm{H}]^{+}$523.25906, found 523.25907; calcd for $\mathrm{C}_{33} \mathrm{H}_{34} \mathrm{~N}_{2} \mathrm{NaO}_{4}[\mathrm{M}+\mathrm{Na}]^{+}$545.24092, found 545.24093; calcd for $\mathrm{C}_{33} \mathrm{H}_{34} \mathrm{KN}_{2} \mathrm{O}_{4}[\mathrm{M}+\mathrm{K}]^{+}$561.21483, found 561.21485.

$\mathrm{N}$-((Cyclohexylcarbamoyl)(2,4,6-trimethylphenyl)methyl)2-oxo-N-phenyl-2H-chromone-3-carboxamide (5h): Yield $90 \% . \mathrm{Mp}=251-254^{\circ} \mathrm{C} . \mathrm{IR}\left(\mathrm{KBr}, \mathrm{cm}^{-1}\right): 3275,1721,1652$ $\mathrm{cm}^{-1} .{ }^{1} \mathrm{H} \mathrm{NMR}\left(300 \mathrm{MHz}, \mathrm{CDCl}_{3}\right): \delta=1.08-2.04(\mathrm{~m}, 10 \mathrm{H}$, $\left.5 \mathrm{CH}_{2}\right), 2.21(\mathrm{~s}, 9 \mathrm{H}, 3 \mathrm{Me}), 3.86(\mathrm{~m}, 1 \mathrm{H}, \mathrm{CH}-\mathrm{NH}), 5.86$ (br s, $1 \mathrm{H}, \mathrm{NH}), 6.21(\mathrm{~s}, 1 \mathrm{H}, \mathrm{CH}-\mathrm{N}), 6.81-7.78(\mathrm{~m}, 10 \mathrm{H}, \mathrm{H}-\mathrm{Ar})$, $7.80(\mathrm{~s}, 2 \mathrm{H},=\mathrm{CH}$ and $\mathrm{H}-\mathrm{Ar}) .{ }^{13} \mathrm{C} \mathrm{NMR}\left(75 \mathrm{MHz}, \mathrm{CDCl}_{3}\right)$

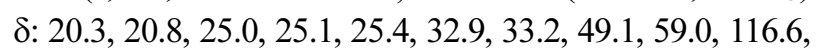

118.0, 124.6, 126.6, 127.9, 128.3, 129.7, 131.1, 131.8, 132.1, $137.5,138.4,139.8,141.8,153.6,157.8,165.5,169.0$. HRMS (ESI) calcd for $\mathrm{C}_{33} \mathrm{H}_{35} \mathrm{~N}_{2} \mathrm{O}_{4}[\mathrm{M}+\mathrm{H}]^{+}$523.25911, found 523.25911; calcd for $\mathrm{C}_{33} \mathrm{H}_{34} \mathrm{~N}_{2} \mathrm{NaO}_{4}[\mathrm{M}+\mathrm{Na}]^{+}$545.24100, found 545.24101; calcd for $\mathrm{C}_{33} \mathrm{H}_{34} \mathrm{KN}_{2} \mathrm{O}_{4}[\mathrm{M}+\mathrm{K}]^{+}$561.21492, found 561.21493.

N-((Cyclohexylcarbamoyl)(2-nitrophenyl)methyl)-2-oxoN-phenyl-2H-chromone-3-carboxamide (5i): Yield 75\%. $\mathrm{Mp}=78-81{ }^{\circ} \mathrm{C} . \mathrm{IR}\left(\mathrm{KBr}, \mathrm{cm}^{-1}\right): 3331,1707,1654,1607 \mathrm{~cm}^{-1}$. ${ }^{1} \mathrm{HNMR}\left(300 \mathrm{MHz}, \mathrm{CDCl}_{3}\right): \delta=1.29-2.10\left(\mathrm{~m}, 10 \mathrm{H}, 5 \mathrm{CH}_{2}\right)$, 3.94 (m, 1H, CH-NH), 7.00-7.90 (m, 15H, CH-N, NH and $\mathrm{H}-\mathrm{Ar}), 8.0(\mathrm{~s}, 1 \mathrm{H},=\mathrm{CH}) .{ }^{13} \mathrm{C} \mathrm{NMR}\left(75 \mathrm{MHz}, \mathrm{CDCl}_{3}\right)$ $\delta: 24.9,25.0,25.4,32.5,33.1,49.3,61.7,116.7,117.9,124.6$, 125.1, 125.6, 128.3, 128.6, 128.7, 129.2, 129.3, 132.7, 132.8, 133.0, 138.1, 143.2, 149.6, 153.6, 158.7, 165.2, 166.9. HR-MS (ESI) calcd for $\mathrm{C}_{30} \mathrm{H}_{28} \mathrm{~N}_{3} \mathrm{O}_{6}[\mathrm{M}+\mathrm{H}]^{+} 526.19762$, found 526.19759; calcd for $\mathrm{C}_{30} \mathrm{H}_{27} \mathrm{~N}_{3} \mathrm{NaO}_{6}[\mathrm{M}+\mathrm{Na}]^{+}$548.17946, found 548.17944; calcd for $\mathrm{C}_{30} \mathrm{H}_{27} \mathrm{KN}_{3} \mathrm{O}_{6}[\mathrm{M}+\mathrm{K}]^{+}$564.15325, found 564.15324 .

N-((Cyclohexylcarbamoyl)(3-nitrophenyl)methyl)-2-0xoN-phenyl-2H-chromone-3-carboxamide (5j): Yield 80\%. $\mathrm{Mp}=212-215^{\circ} \mathrm{C} . \mathrm{IR}\left(\mathrm{KBr}, \mathrm{cm}^{-1}\right): 3282,1739,1719,1654$ $\mathrm{cm}^{-1} .{ }^{1} \mathrm{H} \mathrm{NMR}\left(300 \mathrm{MHz}, \mathrm{CDCl}_{3}\right): \delta=1.35-2.14(\mathrm{~m}, 10 \mathrm{H}$, $\left.5 \mathrm{CH}_{2}\right), 3.97(\mathrm{~m}, 1 \mathrm{H}, \mathrm{CH}-\mathrm{NH}), 6.51(1 \mathrm{H}, \mathrm{s}, \mathrm{CH}-\mathrm{N}), 7.07-$ $7.64(\mathrm{~m}, 12 \mathrm{H}, \mathrm{NH}$ and $\mathrm{H}-\mathrm{Ar}), 7.91(\mathrm{~s}, 1 \mathrm{H},=\mathrm{CH}), 8.05$ (dd, $1 \mathrm{H}, J=8.1 \mathrm{~Hz}, \mathrm{H}-\mathrm{Ar}$ ), 8.10 (d, $1 \mathrm{H}, J=1.0 \mathrm{~Hz}, \mathrm{H}-\mathrm{Ar}$ ). ${ }^{13} \mathrm{C} \mathrm{NMR}\left(75 \mathrm{MHz}, \mathrm{CDCl}_{3}\right) \delta: 24.9,25.0,25.4,32.7,33.1$, 49.4, 64.7, 116.8, 117.8, 123.0, 125.1, 125.4, 125.7, 128.6, 128.7, 128.9, 129.1, 129.4, 133.1, 136.4, 136.8, 138.2, 143.2, 147.8, 153.7, 158.6, 165.6, 166.5. HR-MS (ESI) calcd for $\mathrm{C}_{30} \mathrm{H}_{28} \mathrm{~N}_{3} \mathrm{O}_{6}[\mathrm{M}+\mathrm{H}]^{+}$526.19725, found 526.19725; calcd for $\mathrm{C}_{30} \mathrm{H}_{27} \mathrm{~N}_{3} \mathrm{NaO}_{6}[\mathrm{M}+\mathrm{Na}]^{+} 548.17919$, found 548.17919; calcd for $\mathrm{C}_{30} \mathrm{H}_{27} \mathrm{KN}_{3} \mathrm{O}_{6}[\mathrm{M}+\mathrm{K}]^{+}$564.15305, found 564.15306.

N-((Cyclohexylcarbamoyl)(4-nitrophenyl)methyl)-2-oxoN-phenyl-2H-chromone-3-carboxamide (5k): Yield 84\%. $\mathrm{Mp}=225-227^{\circ} \mathrm{C} . \mathrm{IR}\left(\mathrm{KBr} \mathrm{cm}^{-1}\right): 3269,1717,1655,1646 \mathrm{~cm}^{-1}$. ${ }^{1} \mathrm{H} \mathrm{NMR}\left(300 \mathrm{MHz}, \mathrm{CDCl}_{3}\right): \delta=1.03-1.70\left(\mathrm{~m}, 10 \mathrm{H}, 5 \mathrm{CH}_{2}\right)$, 3.59 (m, 1H, CH-NH), $6.31(1 \mathrm{H}, \mathrm{s}, \mathrm{CH}-\mathrm{N}), 7.02$ (br s, $1 \mathrm{H}, \mathrm{NH}), 7.04$ (d, 2H, $J=8.1 \mathrm{~Hz}, \mathrm{H}-\mathrm{Ar}), 7.07$ (d, 1H, $J=$ $8.5 \mathrm{~Hz}, \mathrm{H}-\mathrm{Ar}$ ), 7.18-7.33 (m, 4H, H-Ar), 7.48 (d, 2H, $J=$ $8.1 \mathrm{~Hz}, \mathrm{H}-\mathrm{Ar}), 7.57$ (t, $1 \mathrm{H}, J=7.5 \mathrm{~Hz}, \mathrm{H}-\mathrm{Ar}), 7.70$ (d, 1H, $J=8.5 \mathrm{~Hz}, \mathrm{H}-\mathrm{Ar}), 8.09$ (s, $1 \mathrm{H},=\mathrm{CH}), 8.25$ (d, 1H, $J=7.5$ $\mathrm{Hz}, \mathrm{H}-\mathrm{Ar}) .{ }^{13} \mathrm{C} \mathrm{NMR}\left(75 \mathrm{MHz}, \mathrm{CDCl}_{3}\right) \delta: 24.4,25.1,30.7$, $32.1,48.1,63.2,116.2,117.6,123.0,124.9,125.0,128.2$, 129.1, 130.4, 131.2, 132.9, 138.5, 142.4, 142.8, 146.8, 152.9, 158.3, 164.5, $166.7 \delta \mathrm{C}_{30} \mathrm{H}_{27} \mathrm{~N}_{3} \mathrm{O}_{6}$ (525.19): C, 68.56; H, 5.18; N, 8.00. Found: C, 68.36; H, 5.27; N, 7.87.

N-((Cyclohexylcarbamoyl)(2-(prop-2-ynyloxy)phenyl) methyl)-2-0xo-N-phenyl-2Hchromene-3-carboxamide (5l): 
Yield 85\%. Mp $=195-196^{\circ} \mathrm{C} . \mathrm{IR}\left(\mathrm{KBr} \mathrm{cm}^{-1}\right): 3300,2124$, $1726,1652 \mathrm{~cm}^{-1}$. ${ }^{1} \mathrm{H}$ NMR (300 MHz, acetone): $\delta=1.20$ $1.75\left(\mathrm{~m}, 10 \mathrm{H}, 5 \mathrm{CH}_{2}\right.$, cyclohexyl), $3.19(\mathrm{t}, 1 \mathrm{H}, J=2.3 \mathrm{~Hz}$, $\mathrm{CH}), 3.86(\mathrm{~m}, 1 \mathrm{H}, \mathrm{CH}-\mathrm{NH}), 4.81\left(\mathrm{~d}, 2 \mathrm{H}, J=2.6 \mathrm{~Hz}, \mathrm{CH}_{2}-\right.$ O), $6.55(\mathrm{~s}, 1 \mathrm{H}, \mathrm{CH}-\mathrm{N}), 6.70(\mathrm{t}, 1 \mathrm{H}, J=7.4 \mathrm{~Hz}, \mathrm{H}-\mathrm{Ar})$, 6.90-6.97 (m, 4H, NH and H-Ar), 7.08-7.13 (m, 2H, HAr), 7.21-7.33 (m, 4H, H-Ar), 7.54-7.69 (m, 3H, H-Ar), $7.93(\mathrm{~s}, 1 \mathrm{H},=\mathrm{CH}) .{ }^{13} \mathrm{C}$ NMR $(75 \mathrm{MHz}$, acetone) $\delta: 25.6$, 25.7, 26.3, 33.4, 33.6, 49.5, 56.4, 60.2, 77.4, 79.7, 112.2, $117.1,119.1,121.3,124.5,125.6,128.3,128.6,129.7,130.2$, 131.1, 132.0, 133.3, 140.0, 142.59, 156.6, 169.0. HR-MS (ESI) calcd for $\mathrm{C}_{33} \mathrm{H}_{31} \mathrm{~N}_{2} \mathrm{O}_{5}[\mathrm{M}+\mathrm{H}]^{+}$535.22320, found 535.22316; calcd for $\mathrm{C}_{33} \mathrm{H}_{30} \mathrm{~N}_{2} \mathrm{NaO}_{5}[\mathrm{M}+\mathrm{Na}]^{+}$557.20517, found 557.20510; calcd for $\mathrm{C}_{33} \mathrm{H}_{30} \mathrm{KN}_{2} \mathrm{O}_{5}[\mathrm{M}+\mathrm{K}]^{+}$573.17909, found 573.17901 .

N-((tert-Butylcarbamoyl)(2-(prop-2-ynyloxy)phenyl) methyl)-2-oxo-N-phenyl-2H-chromene-3-carboxamide (5m): Yield $88 \% . \mathrm{Mp}=130-133{ }^{\circ} \mathrm{C}$. IR $\left(\mathrm{KBr} \mathrm{cm}^{-1}\right)$ : 3397, 2113, $1713,1668,1650 \mathrm{~cm}^{-1} .{ }^{1} \mathrm{H} \mathrm{NMR}\left(300 \mathrm{MHz}, \mathrm{CDCl}_{3}\right): \delta=$ 1.46 (s, 9H, $t$-but), 2.51 (t, $1 \mathrm{H}, J=2.2 \mathrm{~Hz}, \mathrm{CH}), 4.60$ (d, $2 \mathrm{H}$, $J=2.2 \mathrm{~Hz}, \mathrm{CH}_{2}-\mathrm{O}$ ), 6.47 (s, $\left.1 \mathrm{H}, \mathrm{CH}-\mathrm{N}\right), 6.57$ (br s, $1 \mathrm{H}$, $\mathrm{NH}), 6.78-6.83$ (m, 2H, H-Ar), 6.96-6.97 (m, 3H, H-Ar), 7.12-7.26 (m, 6H, H-Ar), 7.39-7.48 (m, 2H, H-Ar), 7.77 $(\mathrm{s}, 1 \mathrm{H},=\mathrm{CH}) .{ }^{13} \mathrm{C} \mathrm{NMR}\left(75 \mathrm{MHz}, \mathrm{CDCl}_{3}\right) \delta: 28.7,51.8$, 55.8, 60.8, 111.4, 116.7, 118.1, 121.2, 123.1, 124.7, 126.2, 127.8, 128.1, 128.4, 129.6, 131.4, 132.3, 139.0, 141.1, 153.6, 155.4, 158.2, 165.3, 168.4. HR-MS (ESI) calcd for $\mathrm{C}_{31} \mathrm{H}_{29} \mathrm{~N}_{2} \mathrm{O}_{5}$ $[\mathrm{M}+\mathrm{H}]^{+}$509.20763, found 509.20758; calcd for $\mathrm{C}_{31} \mathrm{H}_{28} \mathrm{~N}_{2} \mathrm{NaO}_{5}$ $[\mathrm{M}+\mathrm{Na}]^{+}$531.18953, found 531.18949; calcd for $\mathrm{C}_{31} \mathrm{H}_{28} \mathrm{KN}_{2} \mathrm{O}_{5}$ $[\mathrm{M}+\mathrm{K}]^{+}$547.16356, found 547.16351.

N-((Cyclohexylcarbamoyl)(2-(prop-2-ynyloxy)phenyl) methyl)-N-(4-chlorophenyl)-2-oxo-2H-chromene-3-carboxamide (5n): Yield $85 \% . \mathrm{Mp}=195-196^{\circ} \mathrm{C}$. IR $\left(\mathrm{KBr} \mathrm{cm}^{-1}\right)$ : 3338, 2123, 1733, 1674, $1633 \mathrm{~cm}^{-1}$. ${ }^{1} \mathrm{H}$ NMR $(300 \mathrm{MHz}$, $\left.\mathrm{CDCl}_{3}\right): \delta=1.15-2.10\left(\mathrm{~m}, 10 \mathrm{H}, 5 \mathrm{CH}_{2}\right.$, cyclohexyl), 2.53 (s, 1H, CH), 3.93 (m, 1H, CH-NH), 4.67 (s, 2H, $\left.\mathrm{CH}_{2}-\mathrm{O}\right)$, $6.59(\mathrm{~s}, 1 \mathrm{H}, \mathrm{CH}-\mathrm{N}), 6.77-6.90(\mathrm{~m}, 5 \mathrm{H}, \mathrm{NH}$ and $\mathrm{H}-\mathrm{Ar})$, 7.08-7.28 (m, 6H, H-Ar), 7.43-7.53 (m, 2H, H-Ar), 7.84 $(\mathrm{s}, 1 \mathrm{H},=\mathrm{CH}) .{ }^{13} \mathrm{C} \mathrm{NMR}\left(75 \mathrm{MHz}, \mathrm{CDCl}_{3}\right) \delta: 24.9,25.0$, 25.4, 32.7, 33.1, 49.0, 55.8, 60.1, 111.5, 116.8, 118.0, 121.3, 122.6, 124.9, 125.9, 128.3, 128.5, 131.1, 131.5, 132.7, 133.7, 137.2, 142.5, 153.7, 155.4, 158.3, 165.1, 168.1. HR-MS (ESI) calcd for $\mathrm{C}_{33} \mathrm{H}_{30} \mathrm{ClN}_{2} \mathrm{O}_{5}[\mathrm{M}+\mathrm{H}]^{+} 569.18441$, found 569.18435; calcd for $\mathrm{C}_{33} \mathrm{H}_{29} \mathrm{ClN}_{2} \mathrm{NaO}_{5}[\mathrm{M}+\mathrm{Na}]^{+} 591.16612$, found 591.16608; calcd for $\mathrm{C}_{33} \mathrm{H}_{29} \mathrm{ClKN}_{2} \mathrm{O}_{5}[\mathrm{M}+\mathrm{K}]^{+}$ 607.14036, found 607.14030.

N-((tert-Butylcarbamoyl)(2-(prop-2-ynyloxy)phenyl) methyl)-N-(4-chlorophenyl)-2-oxo-2H-chromene-3-carboxamide (5o): Yield $78 \% . \mathrm{Mp}=97-100^{\circ} \mathrm{C}$. IR $\left(\mathrm{KBr} \mathrm{cm}^{-1}\right)$ :
3359, 3298, 2119, 1723, 1654, $1607 \mathrm{~cm}^{-1} .{ }^{1} \mathrm{H}$ NMR (300 $\mathrm{MHz}, \mathrm{CDCl}_{3}$ ): $\delta=1.43$ (s, 9H, $t$-but), 2.53 (t, $1 \mathrm{H}, J=2.3$ $\mathrm{Hz}, \mathrm{CH}$ ), 4.67 (d, 2H, J=2.3 Hz, $\mathrm{CH}_{2}-\mathrm{O}$ ), 6.35 (br s, $1 \mathrm{H}$, $\mathrm{NH}), 6.44$ (s, 1H, CH-N), 6.79-6.85 (m, 2H, H-Ar), 6.93 (d, 2H, $J=8.6 \mathrm{~Hz}, \mathrm{H}-\mathrm{Ar}), 7.11-7.26$ (m, 6H, H-Ar), 7.42 (dd, $1 \mathrm{H}, J=7.2,1.4 \mathrm{~Hz}, \mathrm{H}-\mathrm{Ar}), 7.49$ (dt, $1 \mathrm{H}, J=7.8,1.4 \mathrm{~Hz}$, $\mathrm{H}-\mathrm{Ar}), 7.78(\mathrm{~s}, 1 \mathrm{H},=\mathrm{CH}) .{ }^{13} \mathrm{C} \mathrm{NMR}\left(75 \mathrm{MHz}, \mathrm{CDCl}_{3}\right) \delta$ : 28.7, 51.9, 55.7, 60.5, 111.6, 116.7, 118.0, 121.3, 122.8, 124.8, 125.9, 128.3, 128.4, 130.0, 131.1, 131.3, 132.5, 133.6, 137.4, 1342.2, 153.7, 155.3, 158.0, 165.1, 168.3. HR-MS (ESI) calcd for $\mathrm{C}_{31} \mathrm{H}_{28} \mathrm{CIN}_{2} \mathrm{O}_{5}[\mathrm{M}+\mathrm{H}]^{+}$543.16869, found 543.16864; calcd for $\mathrm{C}_{31} \mathrm{H}_{27} \mathrm{ClN}_{2} \mathrm{NaO}_{5}[\mathrm{M}+\mathrm{Na}]^{+} 565.15050$, found 565.15046; calcd for $\mathrm{C}_{31} \mathrm{H}_{27} \mathrm{ClKN}_{2} \mathrm{O}_{5}[\mathrm{M}+\mathrm{K}]^{+}$581.12444, found 581.12440 .

N-((Cyclohexylcarbamoyl)(2-(prop-2-ynyloxy)phenyl) methyl)-N-(2,4-dichlorophenyl)-2-oxo-2H-chromene-3-carboxamide (5p): Yield 75\%. $\mathrm{Mp}=177-180^{\circ} \mathrm{C} . \mathrm{IR}\left(\mathrm{KBr} \mathrm{cm}^{-1}\right)$ : 3338, 2123, 1733, 1674, $1633 \mathrm{~cm}^{-1}$. ${ }^{1} \mathrm{H}$ NMR (300 MHz, $\left.\mathrm{CDCl}_{3}\right): \delta=1.28-2.16\left(\mathrm{~m}, 10 \mathrm{H}, 5 \mathrm{CH}_{2}\right.$, cyclohexyl), 2.61 (t, $1 \mathrm{H}, J=2.3 \mathrm{~Hz}, \mathrm{CH}), 3.95$ (m, 1H, CH-NH), 4.84 (d, 2H, $\left.J=2.3 \mathrm{~Hz}, \mathrm{CH}_{2}-\mathrm{O}\right), 6.87(\mathrm{~s}, 1 \mathrm{H}, \mathrm{CH}-\mathrm{N}), 6.99$ (d, $1 \mathrm{H}, J=$ $2.4 \mathrm{~Hz}, \mathrm{NH}$ ), 7.07 (m, 5H, H-Ar), 7.18-7.27 (m, 3H, H-Ar), 7.46-7.53 (m, 3H, H-Ar), 7.89 (s, 1H, =CH). ${ }^{13} \mathrm{C}$ NMR $\left(75 \mathrm{MHz}, \mathrm{CDCl}_{3}\right)$ \&: 24.9, 52.0, 25.4, 32.7, 33.1, 49.2, 59.8, $60.9,116.8,117.9,125.0,125.8,128.0,128.5,128.6,128.7$, 129.5, 129.6, 130.2, 130.5, 132.1, 132.8, 138.1, 142.6, 151.1, 153.6, 158.6, 165.2, 166.9. HR-MS (ESI) calcd for $\mathrm{C}_{33} \mathrm{H}_{29} \mathrm{Cl}_{2} \mathrm{~N}_{2} \mathrm{O}_{5}$ $[\mathrm{M}+\mathrm{H}]^{+} 603.14562$, found 603.14555; calcd for $\mathrm{C}_{33} \mathrm{H}_{28} \mathrm{Cl}_{2} \mathrm{~N}_{2} \mathrm{NaO}_{5}$ $[\mathrm{M}+\mathrm{Na}]^{+} 625.12762$, found 625.12754 ; calcd for $\mathrm{C}_{33} \mathrm{H}_{28}$ $\mathrm{Cl}_{2} \mathrm{KN}_{2} \mathrm{O}_{5}[\mathrm{M}+\mathrm{K}]^{+}$641.10149, found 641.10142.

\section{RESULTS AND DISCUSSION}

Coumarin-3-carboxylic acid is an efficient starting material for the synthesis of functionalized coumarin skeleton. It appears in a vast range of natural products with high levels of biological activity and also was used for the synthesis of compounds with pharmaceutical activity. ${ }^{30-32}$ Coumarin3-carboxylic acid $\mathbf{3}$ was obtained from the reaction of salicylaldehyde with Meldrum's acid refluxing in water. ${ }^{33}$ Initially, the four-component reaction of benzaldehyde 1a, aniline 2, coumarin-3-carboxylic acid 3, and cyclohexyl isocyanides $4 \mathbf{a}$ were carried out smoothly in methanol at room temperature was selected as model reaction (Scheme 1). The desired product 5a was isolated in $90 \%$ yield (Table 1 , entry 1). Encouraged by this result, we turned our attention to other substituted benzaldehydes, amines and isocyanides. The desired amidated coumarins $\mathbf{5 b}-\mathbf{p}$ was obtained in 75-92\%. 
Table 1. Synthesis of amidated 3-substituted coumarins using Ugi 4-CR

\begin{tabular}{|c|c|c|c|c|c|}
\hline Entry & $\mathrm{Ar}$ & $\mathrm{R}^{1}$ & $\mathrm{R}^{2}$ & Product & Yield (\%)* \\
\hline 1 & $\mathrm{Ph}$ & $\mathrm{Ph}$ & $\mathrm{Cyc}^{* *}$ & $5 \mathbf{5 a}$ & 90 \\
\hline 2 & $2-\mathrm{MeOC}_{6} \mathrm{H}_{4-}$ & $\mathrm{Ph}$ & Cyc & $\mathbf{5 b}$ & 87 \\
\hline 3 & 4- $\mathrm{MeOC}_{6} \mathrm{H}_{4}-$ & $\mathrm{Ph}$ & Cyc & $5 c$ & 92 \\
\hline 4 & $2,4-(\mathrm{MeO})_{2} \mathrm{C}_{6} \mathrm{H}_{3}-$ & $\mathrm{Ph}$ & Cyc & 5d & 93 \\
\hline 5 & $4,5,6-(\mathrm{MeO})_{3} \mathrm{C}_{6} \mathrm{H}_{2-}$ & $\mathrm{Ph}$ & Cyc & $5 e$ & 88 \\
\hline 6 & 4-Me- $\mathrm{C}_{6} \mathrm{H}_{4-}$ & $\mathrm{Ph}$ & Сyc & $5 f$ & 83 \\
\hline 7 & $4-i-\mathrm{pr}_{-} \mathrm{C}_{6} \mathrm{H}_{4-}$ & $\mathrm{Ph}$ & Сус & $5 g$ & 91 \\
\hline 8 & $2,4,6-(\mathrm{Me})_{3} \mathrm{C}_{6} \mathrm{H}_{2-}$ & $\mathrm{Ph}$ & Сус & $5 \mathbf{h}$ & 90 \\
\hline 9 & $2-\mathrm{O}_{2} \mathrm{~N}-\mathrm{C}_{6} \mathrm{H}_{4-}$ & $\mathrm{Ph}$ & Cyc & $5 i$ & 75 \\
\hline 10 & $3-\mathrm{O}_{2} \mathrm{~N}-\mathrm{C}_{6} \mathrm{H}_{4-}$ & $\mathrm{Ph}$ & Сус & $5 \mathbf{j}$ & 80 \\
\hline 11 & $4-\mathrm{O}_{2} \mathrm{~N}-\mathrm{C}_{6} \mathrm{H}_{4-}$ & $\mathrm{Ph}$ & Cyc & $5 \mathbf{k}$ & 77 \\
\hline 12 & 2-(prop-2-ynyloxy)- $\mathrm{C}_{6} \mathrm{H}_{4}-$ & $\mathrm{Ph}$ & Cyc & 51 & 85 \\
\hline 14 & 2-(prop-2-ynyloxy)- $\mathrm{C}_{6} \mathrm{H}_{4}-$ & $\mathrm{Ph}$ & $t$-butyl $* * *$ & $5 \mathrm{~m}$ & 85 \\
\hline 15 & 2-(prop-2-ynyloxy)- $\mathrm{C}_{6} \mathrm{H}_{4}-$ & $4-\mathrm{Cl}-\mathrm{C}_{6} \mathrm{H}_{4-}$ & Сус & $5 n$ & 88 \\
\hline 16 & 2-(prop-2-ynyloxy)- $\mathrm{C}_{6} \mathrm{H}_{4}-$ & $4-\mathrm{Cl}-\mathrm{C}_{6} \mathrm{H}_{4}-$ & $t$-butyl & 50 & 78 \\
\hline 17 & 2-(prop-2-ynyloxy)- $\mathrm{C}_{6} \mathrm{H}_{4}-$ & $2,4-(\mathrm{Cl})_{2} \mathrm{C}_{6} \mathrm{H}_{3-}$ & Сyc & $5 p$ & 75 \\
\hline
\end{tabular}

*Yields of isolated products

**Cyclohexyl isocyanide

$* * *$ tert-butyl isocyanide

It was satisfying to observe that the reaction was complete in 6 hours and in many cases the reaction products precipitate during the reaction and the products were obtained after recrystallization. This approach was used for the synthesis of a chemical library of coumarins. It was found that these conditions were compatible with a range of electronwithdrawing and electron-donating substituents. The conditions were sufficiently mild to be tolerated by a number of functionalities including nitro and methoxy groups. Reaction with 2,4-dimethoxybenzaldehyde led to the highest yield (93\% yields, Table 1, entry 4).

The structures of compounds $\mathbf{5 a} \mathbf{a}-\mathbf{p}$ were deduced from their spectroscopic data and also high-resolution mass spectrometry data (HR-ESI-MS). The ${ }^{1} \mathrm{H}$ NMR spectrum of the products showed a distinguished peak at region $\delta$ 6.21-6.60 ppm for the $-\mathrm{CH}$ protons and also a broad singlet signal at $\delta 5.86-7.02$ ppm for the $-\mathrm{NH}$ moiety. Partial assignments of these resonances are given in the experimental section. The ${ }^{13} \mathrm{C}$ NMR spectrum revealed two distinct peaks at $\delta(C)$ 157-165 ppm for the amide $\mathrm{C}=\mathrm{O}$ groups, and 166$169 \mathrm{ppm}$ for the carbonyl of lactone in coumarin skeleton. $O$-Propargyloxy benzaldehyde was used as starting material for the synthesis of products $\mathbf{5} \mathbf{l}-\mathbf{p}$. We believe that this new functionality based multicomponent reactions have a huge potential in creating new molecules or simplifying the synthesis of existing compounds and the synthesized products have good potential for further reactions. The products $\mathbf{5 l - p}$ could be used as starting material for some reac-

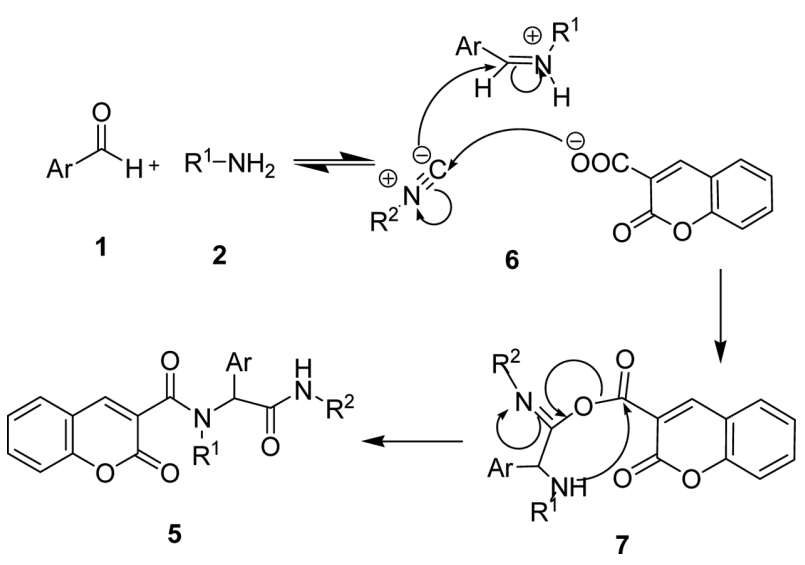

Scheme 2. Proposed mechanism for the synthesis of amidated 3substituted coumarins $\mathbf{5 a}-\mathbf{p}$.

tions such as: Click chemistry, Pauson-Khand reaction, and cyclization reaction.

According to the commonly accepted Ugi-4CR proposed mechanism, it seems that amine, the carbonyl compound and the acid are in equilibrium with the iminiumcarboxylate 6 in the reaction medium. The $\alpha$-addition of the iminium carboxylate onto the carbenoid carbon of the isocyanide leads to the formation of the primary four-component adduct 7 , which undergoes an intramolecular acylation known as Mumm rearrangement to give the stable Ugi adduct 5 (Scheme 2).

Since some of coumarin derivatives could be used as fluorophors, ${ }^{34,35}$ we were interested to investigate the flu- 


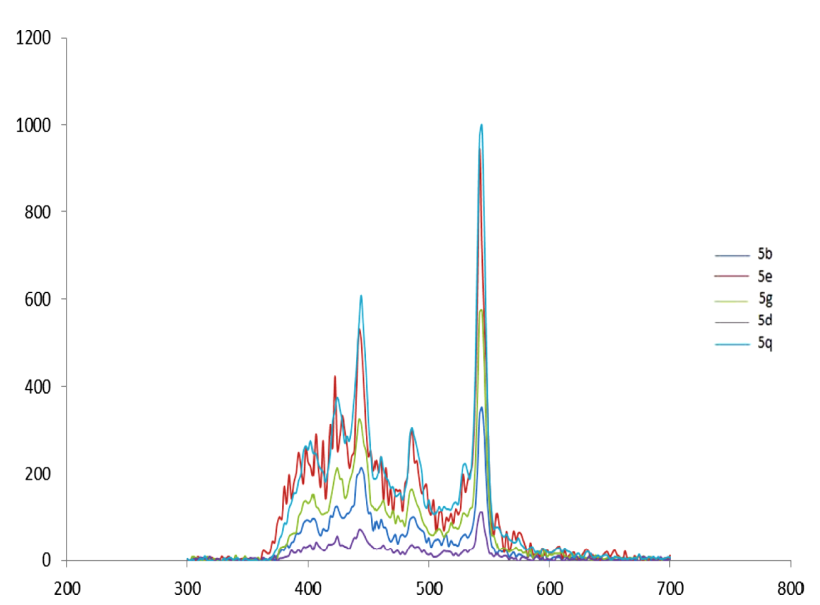

Figure 1. Emission spectra of compounds $5 \mathbf{b}, 5 \mathbf{5 d}, 5 \mathbf{5}, \mathbf{5 g}, \mathbf{5 p}$ in chloform.

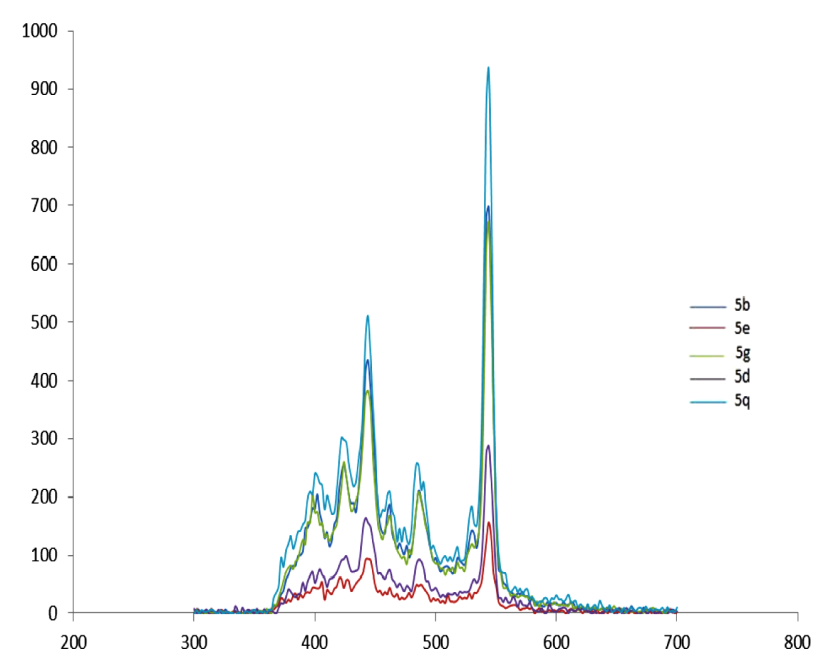

Figure 2. Emission spectra of compounds $5 \mathbf{b}, 5 \mathbf{5 d}, 5 \mathbf{5 e}, \mathbf{5 g}, \mathbf{5 p}$ in acetonitrile.

orescence property of the synthesized compounds. The fluoresence property of some novel amidated coumarines derivatives were investigated in chloroform, acetonitrile, and dimethyl formamide. The wavelengths of the absorption maxima and fluorescence emission maxima fluorescence property of compounds $5 \mathbf{5 b}, \mathbf{5 d}, \mathbf{5 e}, \mathbf{5 g}, \mathbf{5 p}$ were investigated. Excitations were carried out with the four different wavelengths $200,210,220$, and $230 \mathrm{~nm}$, and the emission was investigated in the range $300-700 \mathrm{~nm}$. In the case of 5d, the maximum emission occurred at the excitation wavelength $200 \mathrm{~nm}$, and the maximum emission occurred at the wavelength 542, 546, and $543 \mathrm{~nm}$, respectively (Figs. 1-3). All of compounds displayed almost similar fluorescence emission in the range of $535-547 \mathrm{~nm}$, and they could show a significant difference to coumarin-3-carboxylic acid emission spectra.

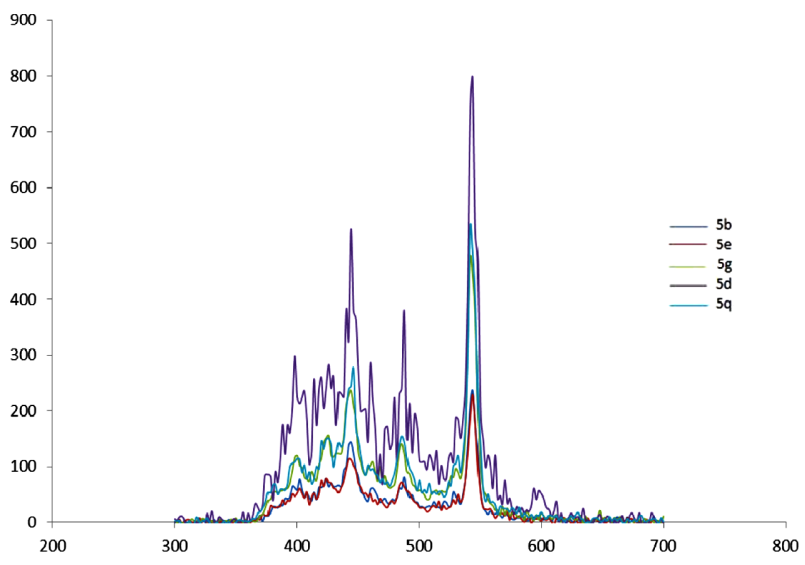

Figure 3. Emission spectra of compounds $5 \mathrm{~b}, 5 \mathrm{~d}, 5 \mathrm{e}, 5 \mathrm{~g}, 5 \mathrm{p}$ in dimethyl formamide.

\section{CONCLUSION}

In conclusion, we have synthesized a small library of 3substituted coumarin carboxamides or pseudopeptides using Ugi-4CR of coumarin-3-carboxylic acid, benzaldehydes, anilines and isocyanides. Some of these novel derivatives could be used for further reactions and have fluorescent spectra in the range $535-547 \mathrm{~nm}$. The simplicity of the synthetic protocol and availability of diverse starting materials make this an attractive strategy for obtaining new coumarins via combinatorial techniques. Investigations of biological activity of these compounds are in progress.

Acknowledgments. Publication cost of this paper was supported by the Korean Chemical Society.

\section{REFERENCES}

1. Murray, R. D. H.; Mendez, J.; Brown, S. A. The Natural Coumarins: Occurrence, Chemistry and Biochemistry; John Wiley: Chichester, U. K., 1982.

2. Keating, G. J.; ÓKennedy, R. In Coumarins: Biology, Applications and Mode of Action; O'Kennedy, R., Thornes, R. D., Eds.; John Wiley and Sons: New York, 1997; pp 23-66.

3. Murray, R. D. H. Natu. Prod. Rep. 1989, 591.

4. Zabradnik, M. The Production and Application of Fluorescent Brightening Agents; John Wiley and Sons: New York, 1992.

5. Maeda, M. Laser Dyes; Academic Press: New York, 1994.

6. Maxwell, A. Mol. Microbiol. 1993, 9, 681.

7. Dexeus, F. H.; Logothetis, C. J.; Sella, A.; Fitz, K.; Amato, R.; Reuben, J. M.; Dozier, N. J. Clin. Oncol. 1990, 8, 325.

8. Zembower, D. E.; Liao, S. Y.; Flavin, M. T.; Xu, Z. Q.; Stup, T. L.; Buckheit, R. W. J. Med. Chem. 1997, 40, 1005. 9. Yu, D.; Suzuki, M.; Xie, L.; Morris-Natschke, S. L.; Lee, 
K. H. Med. Res. Rev. 2003, 23, 322.

10. Kempen, I.; Papapostolou, D.; Thierry, N.; Pochet, L.; Counerotte, S.; Masereel, B.; Foidart, J.-M.; Reboud-Ravaux, M.; Noel, A.; Pirotte, B. Br. J. Cancer 2003, 88, 1111.

11. Finn, G.; Creaven, B.; Egan, D. Eur. J. Pharmacol. 2003, 481,159

12. Finn, G. J.; Creaven, B. S.; Egan, D. A. Cancer Lett. 2004, $205,69$.

13. Morita, H.; Dota, T.; Kobayashi, J. Bioorg. Med. Chem. Lett. 2004, 14, 3665.

14. Kontogiorgis, C. A.; Hadjipavlou-Litina, D. J. Enzyme Inhib. Med. Chem. 2003, 18, 63.

15. Donnelly, A. C.; Mays, J. R.; Burlison, J. A.; Nelson, J. T.; Vielhauer, G.; Holzbeierlein, J.; Blagg, B. S. J. J. Org. Chem. 2008, 73, 8901 .

16. Chimenti, F.; et al. Bioorg. Med. Chem. Lett. 2010, 20, 4922.

17. Bylov, I. E.; Vasylyev, M. V.; Bilokin, Y. V. Eur. J. Med. Chem. 1999, 34, 997.

18. Kempen, I.; et al. Eur. J. Med. Chem. 2008, 43, 2735.

19. Reddy, N. S.; Gumireddy, K.; Mallireddigari, M. R.; Cosenza, S. C.; Venkatapuram, P.; Bell, S. C.; Reddy, E. P.; Reddy, M. V. R. Bioorg. Med. Chem. 2005, 13, 3141.

20. Melagraki, G.; Afantitis, A.; Igglessi-Markopoulou, O.; Detsi, A.; Koufaki, M.; Kontogiorgis, C.; Hadjipavlou-Litina, D. J. Eur. J. Med. Chem. 2009, 44, 3020.

21. Jung, H. S.; Ko, K. C.; Kim, G.-H.; Lee, A.-R; Na, Y.-C.; Kang, C.; Lee, J. Y.; Kim, J. S. Org. Lett. 2011, 13, 1498.

22. Yu, T.; Zhang, P.; Zhao, Y.; Zhang, H.; Meng, J.; Fan, D. Org. Elect. 2009, 10, 653.

23. Katritzky, A. R.; Abdelmajeid, A.; Tala, S. R.; Amine, M. S.; Steel, P. J. Synthesis 2011, 83.

24. Katritzky, A. R.; Ibrahim, T. S.; Tala, S. R.; Abo-Dya, N. E.; Abdel-Samii, Z. K.; El-Feky, S. A. Synthesis 2011, 1494.

25. (a) Zhu, J.; Bienayme, H. Eds. Multicomponent Reactions; Wiley-VCH: Weinheim, 2005. (b) Tietze, L. F.; Brasche, G.; Gericke, K. M. Domino Reactions in Organic Synthesis; Wiley-VCH: Weinheim, 2006. (c) Orru, R. V. A.; Greef,
M. Synthesis, 2003, 1471. (d) Bienayme, H.; Oddon, C.; Schmidt, P. Chem. Eur. J. 2000, 6, 3321. (e) Schreiber, S. L. Science 2000, 287, 1964.

26. (a) Dömling, A.; Ugi, I. Angew. Chem. Int. Ed. 2000, 39, 3168. (b) Sunderhaus, J. D.; Martin, S. F. Chem. Eur. J. 2009, 15, 1300. (c) Isambert, N.; Lavilla, R. Chem. Eur. J. 2008, 14, 8444. (d) Ruijter, E.; Scheffelaar, R.; Orru, R.V. A. Angew. Chem. Int. Ed. 2011, 50, 6234.

27. (a) Dömling, A. Chem. Rev. 2006, 106, 17. (b) Tietze, L. F.; Brasche, G.; Gericke, K. M. In Domino Reactions in Organic Synthesis; Wiley-VCH, 2006. (c) Dömling, A.; Wang, W.; Wang, K. Chem. Rev. 2012, 112, 3083.

28. Che, C.; Li, S.; Jiang, X.; Quan, J.; Lin, S.; Yang, Z. Org. Lett. 2010, 12, 4682.

29. (a) Bararjanian, M.; Balalaie, S.; Rominger, F.; Movassagh, B.; Bijanzadeh. H. R. J. Org. Chem. 2010, 75, 2806. (b) Bararjanian, M.; Hosseinzadeh, S.; Balalaie, S.; Bijanzadeh. H. R. Tetrahedron 2011, 67, 2644. (c) Bararjanian, M.; Balalaie, S.; Rominger, F.; Barouti, S. A. Helv. Chim. Acta. 2010, 93, 777. (d) Khoshkholgh, M. J.; Lotfi, M.; Balalaie, S.; Rominger, F. Tetrahedron 2009, 65, 4228. (e) Bararjanian, M.; Hosseinzadeh, S.; Balalaie, S.; Bijanzadeh, H. R.; Wolf, E. Tetrahedron Lett. 2011, 52, 3329. (f) Khoshkholgh, M. J.; Balalaie, S.; Gleiter, R.; Rominger, F. Tetrahedron 2008, 64, 10924. (g) Ghabraie, E.; Balalaie, S.; Bararjanian, M.; Rominger, F.; Bijanzadeh. H. R. Tetrahedron 2011, 67, 5415.

30. Bhalla, M.; Hitkari, A.; Gujrati, V. R.; Bhalla, T. N.; Shanker, K. Eur. J. Med. Chem. 1994, 29, 713.

31. Bosignore, L.; Cottiglia, F.; Lavagna, S. M.; Loy, G.; Secci, D. Eur. J. Med. Chem. 1994, 29, 479.

32. Bosignore, L.; Cottiglia, F.; Lavagna, S. M.; Loy, G.; Secci, D. Heterocycles 1999, 50, 469.

33. Maggi, R.; Bigi, F.; Carloni, S.; Mazzacani, A.; Sartori, G. Green. Chem. 2001, 3, 173.

34. Key, J. A.; Koh, S.; Timerghazin, Q. K.; Brown, A.; Cairo, C. W. Dyes \& Pigments 2009, 82, 196.

35. Zhao, Y. R.; Zheng, Q.; Dakin, K.; Xu, K.; Martinez, M. L.; Li, W.-H. J. Am. Chem. Soc. 2004, 126, 4653. 